\title{
Silicone Oil-Induced Ocular Hypertension in Mouse Models Glaucomatous
} Neurodegeneration and Neuroprotection

Jie Zhang ${ }^{1,2, \dagger}$, Liang Li ${ }^{1, \dagger}$, Haoliang Huang1, Hannah C. Webber ${ }^{1}$, Pei Zhuang ${ }^{1}$, Liang Liu ${ }^{1}$, Roopa Dalal', Peter H. Tang ${ }^{1,3}$, Vinit B. Mahajan ${ }^{1,3}$, Yang Sun ${ }^{1,3}$, Shaohua Li $^{2}$, Mingchang Zhang $^{2}$, Jeffrey Louis Goldberg ${ }^{1}$ and Yang $\mathrm{Hu}^{1, *}$

${ }^{1}$ Department of Ophthalmology, Stanford University School of Medicine, Palo Alto, CA 94304,

10 USA

$11{ }^{2}$ Department of Ophthalmology, Union Hospital, Tongji Medical College, Huazhong University

12 of Science \& Technology, Wuhan 430022, China

$13{ }^{3}$ Department of Ophthalmology, Veterans Affairs Palo Alto Health Care, Palo Alto, CA 94304,

14 USA

15

16 †These authors contributed equally.

17

$18 *$ Correspondence and requests for materials should be addressed to Y.H.

19 (huyang@stanford.edu). 


\section{Abstract}

Understanding the molecular mechanism of glaucoma and development of neuroprotectants are significantly hindered by the lack of a reliable animal model that accurately recapitulates human glaucoma. Here we sought to develop a mouse model for the secondary glaucoma that is often observed in humans after silicone oil (SO) blocks the pupil or migrates into the anterior chamber following vitreoretinal surgery. We observed similar intraocular pressure (IOP) elevation after intracameral injection into mouse eyes of SO, and removing the SO allows the IOP level to quickly return to normal. This simple, inducible and reversible mouse model showed dynamic changes of visual function that correlate with progressive RGC loss and axon degeneration. We also used a single AAV vector for the first time to co-express miRNA-based shRNA and a neuroprotective transgene and further validated this model as an effective in vivo means to test neuroprotective therapies by targeting neuronal endoplasmic reticulum stress. 


\section{Introduction}

37 Glaucoma is the most common cause of irreversible blindness and will affect more than 100 million individuals between 40 to 80 years of age by 2040(1). Annual direct medical costs to treat this disease in 2 million patients in the United States totaled $\$ 2.9$ billion(2). Glaucoma is a neurodegenerative disease characterized by optic neuropathy with thinning of the retinal nerve fiber layer (RNFL) followed by progressive retinal ganglion cell (RGC) degeneration(3-11). Elevated intraocular pressure (IOP) is the most common risk factor(12). Current therapies target reduction of IOP, but irreversible RGC death continues even after IOP is normalized(13-15), indicating the critical clinical need to prevent degeneration of glaucomatous RGCs and optic nerve (ON). Similar to other chronic neurodegenerative diseases(16), the search for neuroprotectants to treat glaucoma is ongoing. To longitudinally assess the molecular mechanisms of glaucomatous degeneration and the efficacy of neuroprotectants, a reliable, reproducible, and inducible experimental ocular hypertension/glaucoma model is critically important. modeling human diseases and large-scale genetic manipulations. Various rodent ocular hypertension models have been developed including spontaneous mutant or transgenic mice and rats and mice with inducible blockage of aqueous humor outflow from the trabecular meshwork $(\mathrm{TM})(17-20)$. While genetic mouse models are valuable to understand the roles of a specific gene in IOP elevation and/or glaucomatous neurodegeneration, the pathologic effects may take months to years to manifest. Inducible ocular hypertension that develops more quickly and is more severe term would be preferable for experimental manipulation and general mechanism 
3

studies, especially for neuroprotectant screening. Injection of hypertonic saline and laser photocoagulation of the episcleral veins and TM are commonly used in rats and larger animals(18). Although similar techniques also produce ocular hypertension in mice(21-23), they are technically challenging, and irreversible ocular tissue damage and intraocular inflammation complicate their interpretation(17, 20). Intracameral injection of microbeads to occlude aqueous humor circulation through TM produces excellent IOP elevation and glaucomatous neurodegeneration(24-27). However, the difficulty of retaining microbeads at the angle of anterior chamber and of controlling the degree of aqueous outflow blockade results in a low success rate and high variabilities in the magnitude of IOP elevation and neurodegeneration. Furthermore, its lengthy duration (6-12 weeks after microbeads injection) causes death of only $30 \%$ of $\operatorname{RGC}(26,28,29)$, leaving a narrow window for preclinical testing of neuroprotective therapies. It is therefore critically important to develop a simple but effective ocular hypertension model in mice that closely resembles human glaucoma, and that can be readily adapted to larger animals with minimal confounding factors.

4

(1)

operative complication following the intravitreal use of silicone oil (SO) in human vitreoretinal surgery $(30,31)$. SO is used as a tamponade in retinal detachment repair due to it being buoyant with high surface tension. However, SO is lighter than the aqueous and vitreous fluids and an excess can physically occlude the pupil, which in turn prevents aqueous flow into the anterior chamber. This obstruction leads to increased aqueous pressure in the posterior chamber and anterior displacement of the iris, which causes angle-closure, blockage of aqueous outflow through TM, and a further increase in IOP. Based on this clinical experience, we tested a simple 
procedure for intracameral injection of SO to block the pupil and cause ocular hypertension, which faithfully replicates post-operative secondary glaucoma. when the ER, the organelle responsible for synthesis and proper folding of proteins, is overwhelmed by unfolded and misfolded proteins, a process that is called the unfolded protein response $(\mathrm{UPR})(32,33)$. We previously identified the important role of ER stress in glaucomatous neurodegeneration and demonstrated significant neuroprotection of RGC and ON by modulating ER stress molecules, C/EBP homologous protein (CHOP) and X-box binding protein 1 (XBP-1)(29, 34, 35). Here we apply an adeno-associated virus (AAV) that mediates CHOP inhibition and XBP-1 activation in a single vector to the SO-induced ocular hypertension model. We report that this treatment increases RGC somata and axon survival and significantly improves recovery of visual function, which validates these two ER stress molecules as therapeutic targets for glaucoma neuroprotection. This proof-of-concept study demonstrates this model to be an effective way to test neuroprotective strategies in vivo, which can be adapted more broadly to larger pre-clinical animals. 


\section{Results}

Intracameral SO injection induces ocular hypertension by blocking the pupil and aqueous humor drainage in the mouse eyes

Although intravitreal injection of $\mathrm{SO}$ in vitreoretinal surgeries can cause post-operative secondary glaucoma in humans $(30,31)$, we reasoned that direct injection of SO into the anterior chamber of mice would be more efficient, preventing the need to remove the vitreous and reducing toxicity due to direct contact with the retina. As illustrated in Fig. 1A,B and Movie S1, after intracameral injection SO forms a droplet in the anterior chamber that contacts the surface of the iris and tightly seals the pupil due to high surface tension. To test whether SO blocks migration of liquid from the back of the eye to the anterior chamber, we injected dye (DiI) into the posterior chamber and visualized its migration into the anterior chamber. In dramatic contrast to a normal naïve eye, in which copious dye passed through the pupil and appeared in the anterior chamber almost immediately after injection, no injected dye reached the anterior chamber of the SO eye (Movie S2,S3). This result indicates that SO causes effective pupillary block.

The ciliary body constantly produces aqueous humor, which accumulates in the posterior chamber and pushes the iris forward. When the iris root touches the posterior corneal surface, the anterior chamber angle closes (Fig. 1A), as evidenced by live anterior chamber optical coherence tomography (OCT) (Fig. 1B). The angle closure can further impede the outflow of aqueous humor through TM and also contributes to IOP elevation. Dilation of the pupil until it is larger than the SO droplet can relieve the pupillary block. Movie S4 shows that after pupil dilation aqueous humor floods into the anterior chamber and pushes the SO droplet away from the iris, which reopens the anterior chamber angle $($ Fig. 1A,B). Together, these results characterize the 
bioRxiv preprint doi: https://doi.org/10.1101/547661; this version posted February 13 2019. The copyright holder for this preprint (which was not certified by peer review) is the author/funder, who has granted bioRxiv a license to display the preprint in perpetuity. It is made available under aCC-BY 4.0 International license.

122 series of reactions initiated by intracameral SO injection, including the physical mechanisms of

123 SO-induced pupillary block, posterior accumulation of aqueous humor, peripheral angle-closure,

124 and IOP elevation. 
A Corneal tunnel incision and SO injection

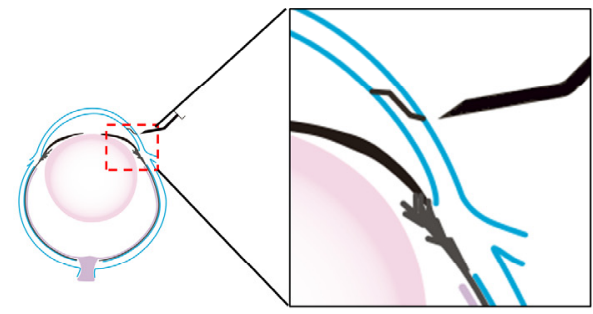

Angle closure due to IOP elevation

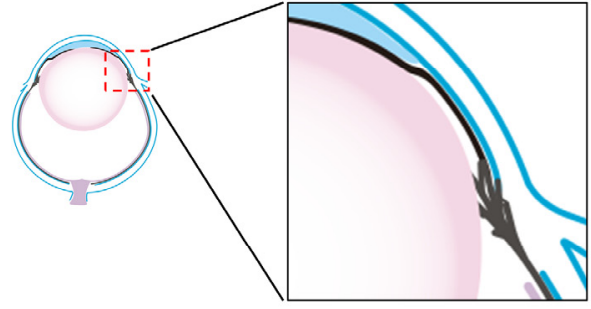

Angle reopen due to aqueous humor infiltration after pupil dilation

C
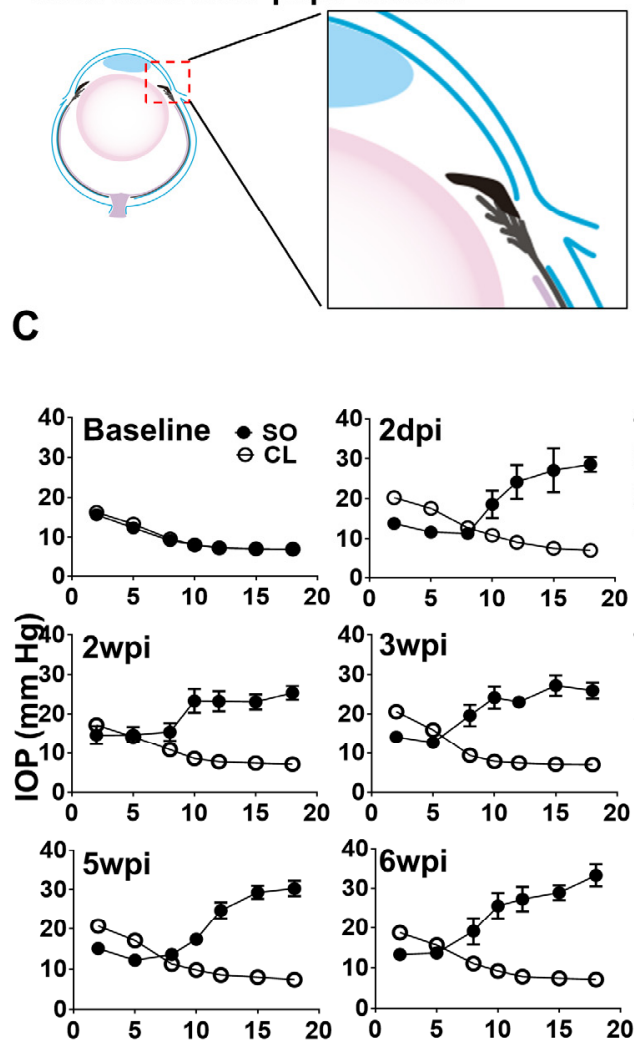

125

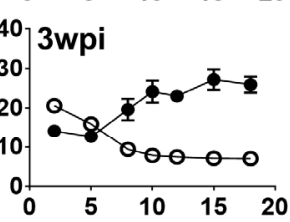

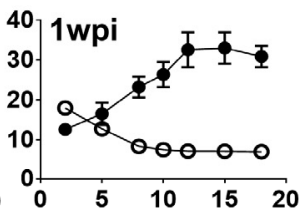
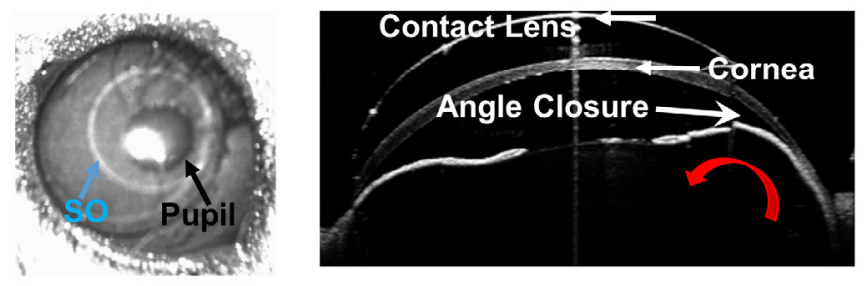

Pupil Dilation
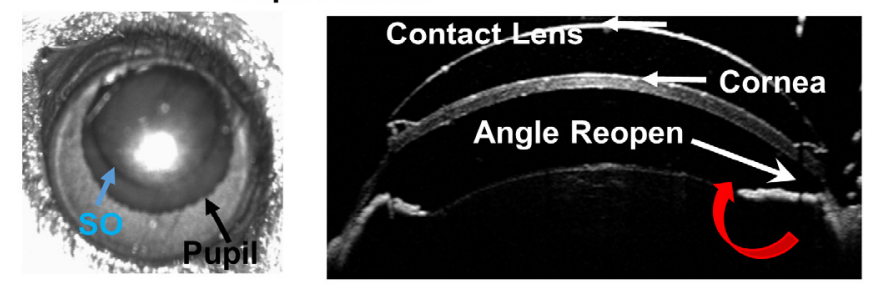

D

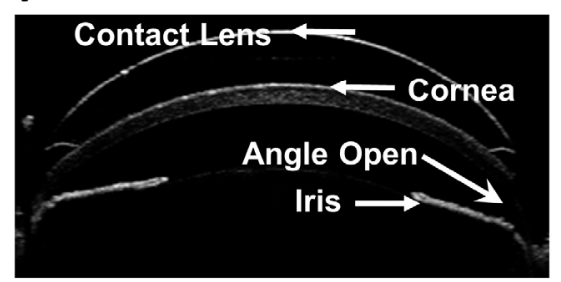

After Injection
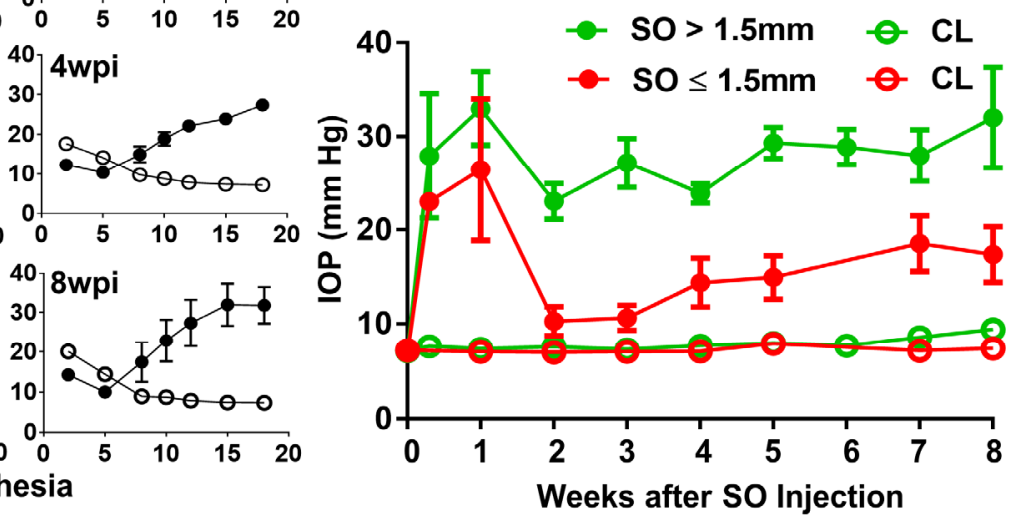

Figure 1. Silicone oil-induced ocular hypertension under-detected (SOHU) mouse model.

127 (A) Cartoon illustration of SO intracameral injection, pupillary block, closure of the anterior 

Representative anterior chamber OCT images of SOHU eyes in living animals showing the relative size of SO droplet (blue arrow) to pupil (black arrow) and the corresponding closure or opening of the anterior chamber angle before and after pupil dilation. Red curved arrow indicates the direction of aqueous humor flow. (C) Longitudinal IOP measurements at different time points before and after SO injection, and continuous measurements for 18 minutes after anesthesia with isoflurane at each time point. (D) The sizes of SO droplet and corresponding IOP measurements at different time points after SO injection; IOP measured 12-15 minutes after anesthesia. SO: SO injected eyes; $\mathrm{CL}$ : contralateral control eyes. $\mathrm{SO}>1.5 \mathrm{~mm}, \mathrm{n}=17$; $\mathrm{SO} \leq$ $1.5 \mathrm{~mm}, \mathrm{n}=6$. contralateral control (CL) eyes after a single normal saline injection once weekly for 8 weeks.

141 Surprisingly, IOP was lower in the SO eyes than in CL eyes when measured immediately after anesthetizing the animals with isoflurane (Fig. 1C). The TonoLab tonometer used to measure

143 mouse IOP is based on a rebound measuring principle that uses a very light weight probe to make momentary contact with the center of the cornea, which primarily measures the pressure of 145 anterior chamber. Measurements over extended periods of time showed the IOP of the SO eyes 146 to be progressively and significantly elevated, in dramatic contrast to the CL eyes, in which IOP 147 decreased over time. The increasing IOP in the SO eyes closely correlated with the change in 148 pupillary size, indicating a significant role of pupillary block. Pupillary dilation removed the 149 pupillary block and allowed the tonometer to detect true IOP after aqueous humor migration into 150 the anterior chamber. Pupillary size reached its maximum and IOP reached to its plateau about 
12-15 minutes after induction of anesthesia with continuous isoflurane inhalation. Therefore, we standardized the time period (12-15 minute after anesthesia) for measuring IOP in later experiments. Because the unique feature of this novel experimental glaucoma model is that the ocular hypertension is under-detected in non-dilated eyes, we named it "SO-induced ocular hypertension under-detected (SOHU)".

IOP elevation in the SO eye started as early as 2 days post injection (2dpi) and remained stable for at least 8 weeks (the longest time point we tested) at an IOP about 2.5 fold that of CL eyes, if the diameter of the SO droplet was larger than $1.5 \mathrm{~mm}$ (Fig. 1D). We achieved this size of SO droplet in about $80 \%$ of mice, but in the $20 \%$ of mice with a small SO droplet $(\leq 1.5 \mathrm{~mm})$ in the anterior chamber due to poor injection or oil leaking, in which the IOP initially increased but dropped soon afterwards (Fig. 1D). Therefore, by observing the size of the SO droplet, it is possible to identify mice very early that will not show elevated IOP and exclude them from subsequent experiments.

\section{Visual function deficits and dynamic morphological changes in SOHU eyes of living animals}

To determine the dynamic changes in RGC morphology and function in SOHU eyes, we longitudinally measured the thickness of the ganglion cell complex (GCC) by OCT(36), visual acuity by the optokinetic tracking response $(\mathrm{OKR})(37,38)$, and general RGC function by pattern electroretinogram (PERG)(39) in living animals. Clinically, the thickness of the RNFL measured by posterior OCT serves as a reliable biomarker for glaucomatous RGC degeneration(40-42).

Because the mouse RNFL is too thin to be reliably measured, we used the thickness of GCC(36), 
174 including RNFL, ganglion cell layer (GCL) and inner plexiform layer (IPL) together, to monitor

175 degeneration of RGC axons, somata, and dendrites caused by ocular hypertension. GCC in

176 SOHU eyes became gradually and progressively thinner (about 84\%, 65\%, 61\% and 53\% of CL

177 eyes) at $1,3,5$ and 8 weeks post injection (wpi), although GCC thinning was not statistically

178 significant at 1wpi (Fig. 2A,B). These results indicate progressive RGC degeneration in response

179 to IOP elevation in SOHU eyes. 
A

OCT

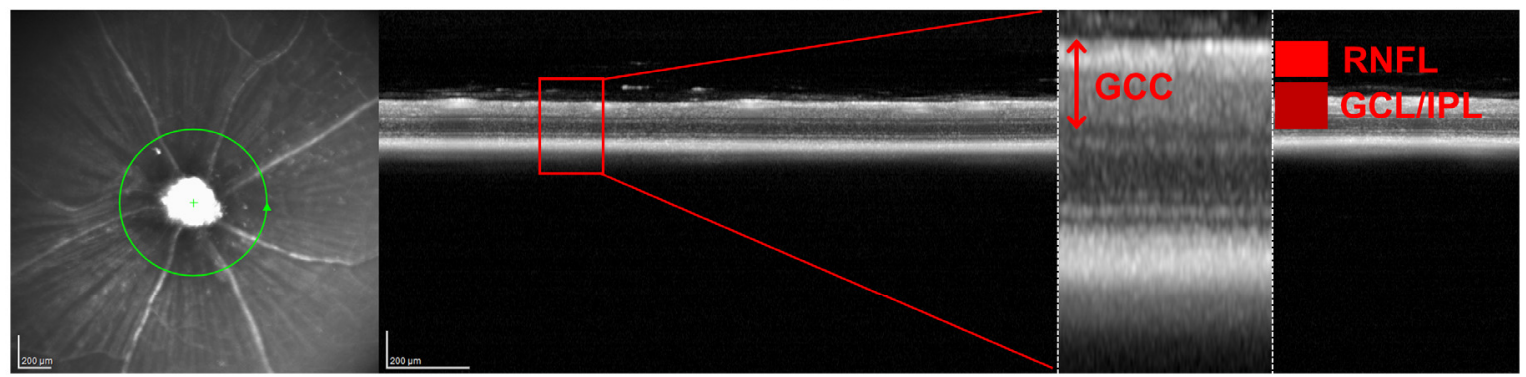

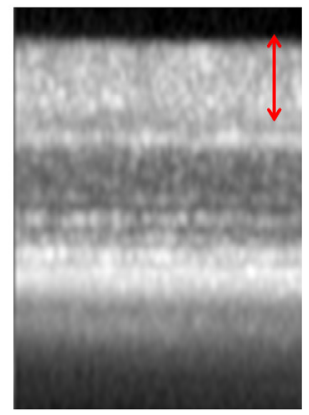

0

B

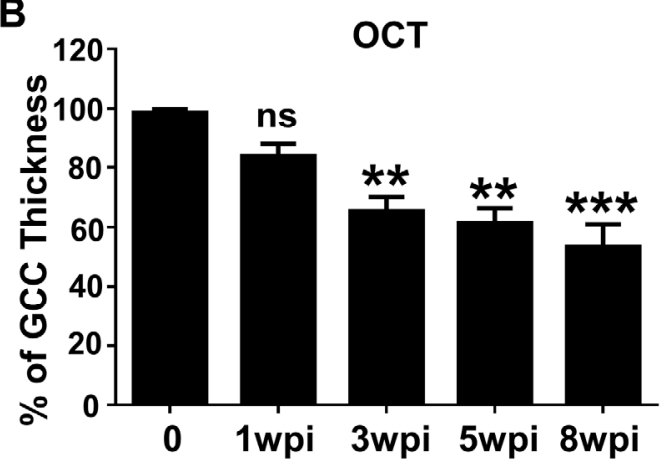

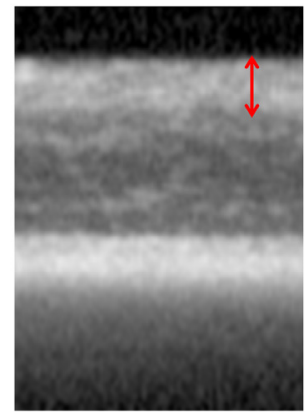

3wpi

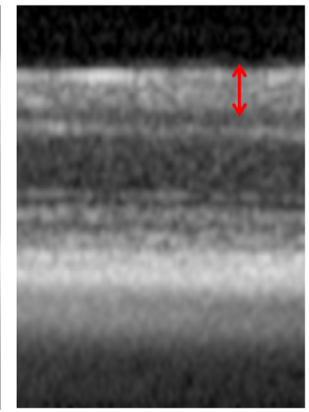

5wpi

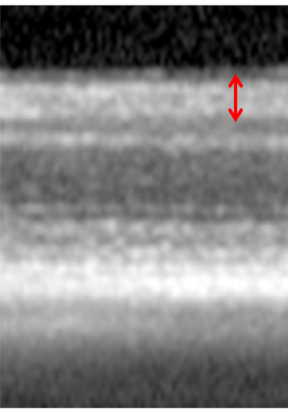

8wpi

C

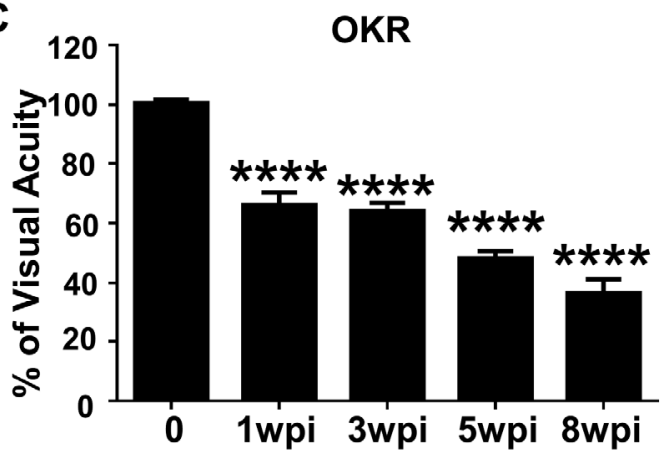

D
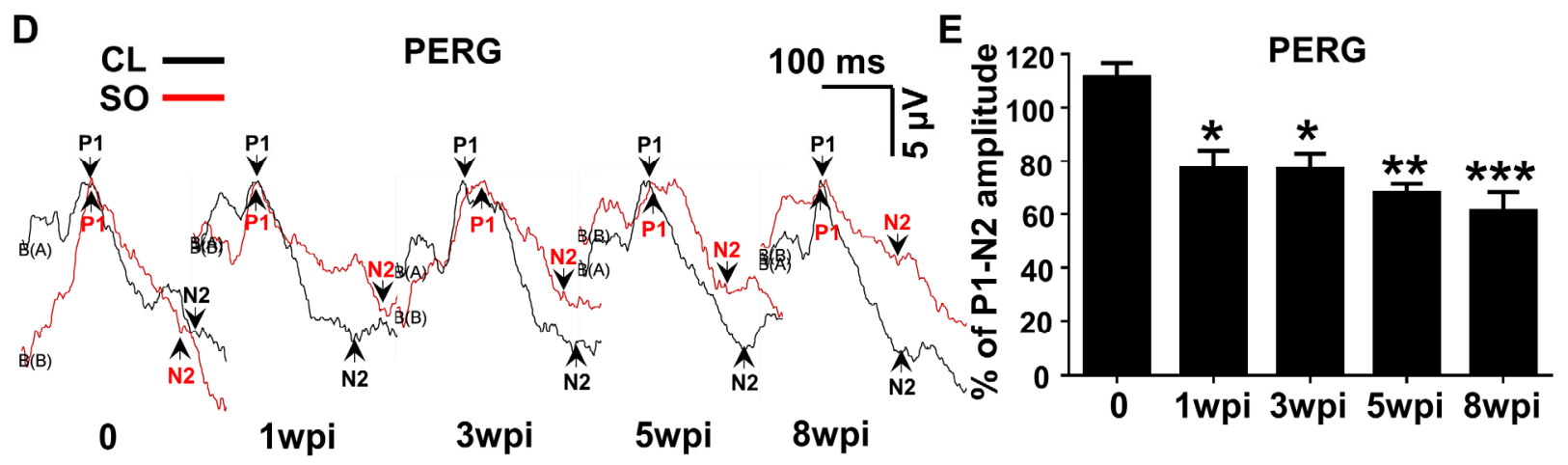

Fiqure 2

181 Figure 2. Dynamic changes in RGC morphology and visual function in living SOHU

182 animals. (A) Representative OCT images of mouse retina. Green circle indicates the OCT scan 
183

184

185

186

187

188

189

190

191

192

193

194

195

196

197

198

199

200

201

202

203

204

205

area surrounding ON head. GCC: ganglion cell complex, including RNFL, GCL and IPL layers; indicated by double end arrows. (B) Quantification of GCC thickness, represented as percentage of GCC thickness in the SO eyes, compared to the CL eyes. $n=10-20$. (C) Visual acuity measured by OKR, represented as percentage of visual acuity in the SO eyes, compared to the CL eyes. $n=10-20$. (D) Representative waveforms of PERG in the contralateral control (CL, black lines) and the SO injected (SO, red lines) eyes at different time points after SO injection. P1: the first positive peak after the pattern stimulus; N2: the second negative peak after the pattern stimulus. (E) Quantification of P1-N2 amplitude, represented as percentage of P1-N2 amplitude in the SO eyes, compared to the CL eyes. $n=13-15$. Data are presented as means \pm s.e.m, *: $\mathrm{p}<0.05, * *: \mathrm{p}<0.01, * * *: \mathrm{p}<0.001, * * * *: \mathrm{p}<0.0001$, one-way ANOVA with Tukey’s multiple comparison test.

.

OKR is a natural reflex that objectively assesses mouse visual acuity $(37,38)$. The mouse eye will only track a grating stimulus that is moving from the temporal to nasal visual field, which allows both eyes to be measured independently $(38,43)$. It has been used to establish correlations between visual deficit and RGC loss in the DBA/2 glaucoma mouse model(44). The visual acuity of SOHU eyes decreased rapidly at 1wpi and stabilized for several more weeks until 5wpi and 8wpi (Fig. 2C). PERG is an important electrophysiological assessment of general RGC function, in which the ERG responses are stimulated with contrast-reversing horizontal bars alternating at constant mean luminance(39). Our PERG system measured both eyes at the same time, so there was an internal control to use as a reference and normalization to minimize the variations. Consistent with visual acuity deficit, the P1-N2 amplitude ratio of the SO eyes to CL eyes decreased progressively (Fig. 2D,E). These results suggest that RGCs are very sensitive 
to IOP elevation, but resilient for a period of time before further degeneration. Taken together, these in vivo results show that SOHU eyes developed progressive structural and visual function deficits that closely resemble changes in glaucoma patients.

\section{Glaucomatous degeneration of RGC somata and axons in SOHU eyes}

211 In vivo functional and imaging results indicate significant neurodegeneration in SOHU eyes, and

212 histological analysis of post-mortem tissue samples supports these findings. We quantified

213 surviving RGC somata in retinal wholemounts and surviving axons in ON semithin cross-

214 sections at multiple time points after SO injection. Similar to the changes of GCC thickness

215 measured by OCT in vivo, there was no statistical significance in surviving RGC counts between

216 SOHU and control eyes at 1wpi, whereas there was significant and worsening RGC loss at 3, 5

217 and 8wpi, when only 43\%, 28\%, and 12\% of RGCs survived (Fig. 3A,B). This result confirmed

218 significant progressive RGC death in response to IOP elevation in SOHU eyes. Significant RGC

219 axon degeneration also occured in SOHU eyes; only 57\%, 41\% and 35\% RGC axons survived at

220 3, 5, and 8wpi (Fig. 3A,C). Therefore, IOP elevation in SOHU mouse eyes produces

221 glaucomatous RGC and $\mathrm{ON}$ degeneration that starts as early as 3 wpi and becomes progressing

222 more severe at later time points that correlate with visual function deficits. 

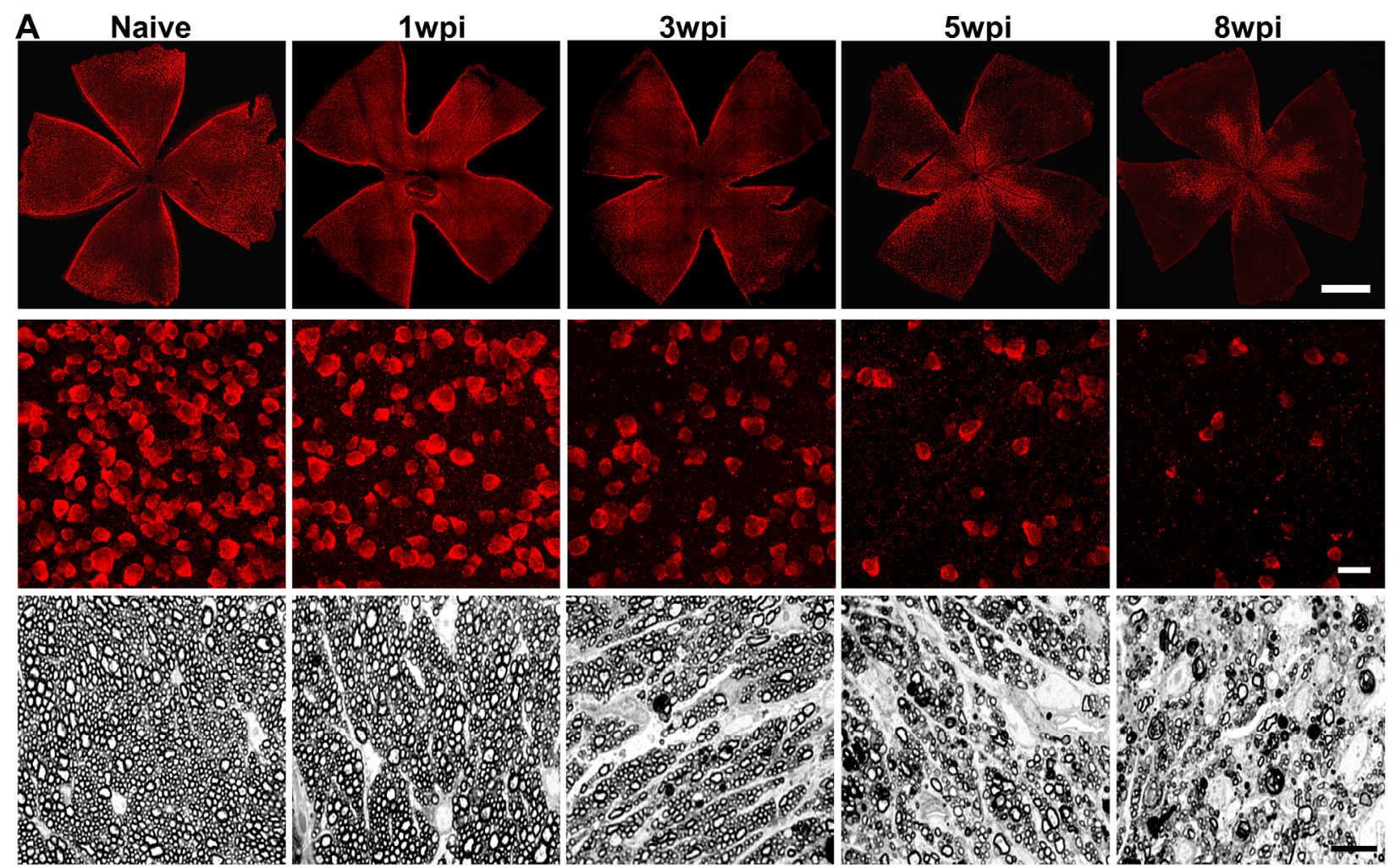

B

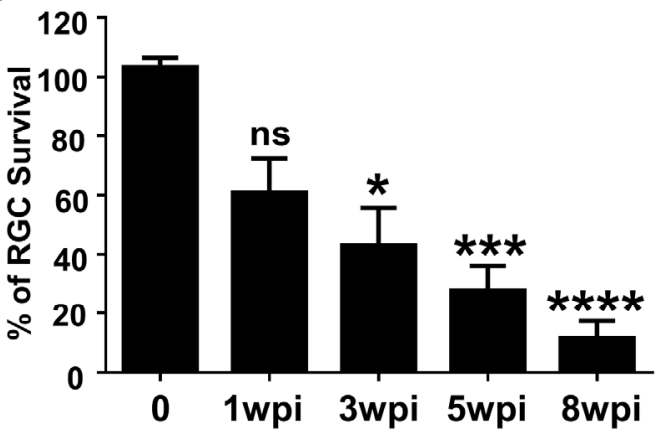

C

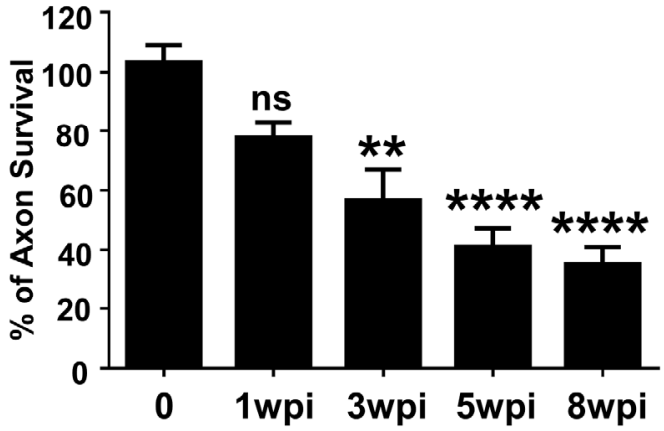

Figure 3

Figure 3. Glaucomatous RGC soma and axon degeneration in SOHU eyes. (A) Upper panel,

228 portion of flat-mounted retinas showing surviving RBPMS-positive (red) RGCs at different time

229 points after SO injection. Scale bar, $20 \mu \mathrm{m}$. Lower panel, light microscope images of semi-thin

230 transverse sections of ON stained with PPD at different time points after SO injection. Scale bar,

$23110 \mu \mathrm{m}$. (B,C) Quantification of surviving RGCs ( $n=11-13)$ and surviving axons in ON $(n=10-16)$ 
232 at different time points after SO injection, represented as percentage of SO eyes compared to CL

eyes. Data are presented as means \pm s.e.m. $* P<0.05, * * P<0.01, * * *: P<0.001, * * * *: P<0.0001$; one-way ANOVA with Tukey's multiple comparison test.

Although the SO used in these studies was sterile and safe for human use, we considered neurodegeneration phenotypes observed in SOHU eyes are glaucomatous responses to ocular hypertension. and protect glaucomatous RGCs and $\mathrm{ON}$ in SOHU eyes

We previously showed that ER stress is induced by ON injury and that CHOP deletion in combination with XBP-1s overexpression synergistically promotes neuroprotection of RGC somata and axons in mouse ON crush model, microbead-induced glaucoma model and EAE/optic neuritis(29, 34, 35). These results suggested that these ER stress molecules are

251 potential therapeutic targets for neuroprotection in optic neuropathies. We therefore examined 252 whether the SOHU model can be used to evaluate the effect of ER stress manipulation on 253 glaucomatous RGCs and ON. We previously demonstrated that the microRNA-based AAV254 CHOP shRNA-GFP knocks down endogenous CHOP in mouse retinas and increases RGC soma 
255 and axon survival in the ON crush model and microbeads-induced glaucoma model(29). We

256 modified this AAV vector by replacing GFP with XBP-1s to knockdown CHOP and express

257 XBP-1s with a single AAV vector. SO injection elevated IOP to a similar degree in mice injected 258 intravitreally with AAV-control shRNA-GFP or AAV-CHOP shRNA-XBP-1s (Fig. 4A), but

259 significant differences in visual acuity and GCC thickness were present at 8wpi (Fig. 4B,C).

260 About $60 \%$ of RGCs and $67 \%$ of axons survived at 8 wpi in SOHU eyes injected with AAV-

261 control shRNA-GFP, whereas approximately $91 \%$ of RGCs and $98 \%$ of axons survived in

262 SOHU eyes treated with AAV-CHOP shRNA-XBP-1s (Fig. 4D,E). We want to point out that

263 transduction of AAV itself produces noticeable neuroprotection compared to SOHU eyes

264 without AAV injection. Although the mechanism is unknown, AAV also provided

265 neuroprotection in other optic neuropathy models that we tested (data not shown). In summary,

266 our proof-of-concept studies demonstrated the effectiveness of AAV-mediated gene therapy and

267 the usefulness of the SOHU model in screening neuroprotectants, in addition to confirming our

268 previous findings that combined inhibition of the PERK-eIF2 $\alpha$-CHOP branch of ER stress and

269 activation of the XBP-1 pathway is a promising therapeutic approach for glaucoma. 

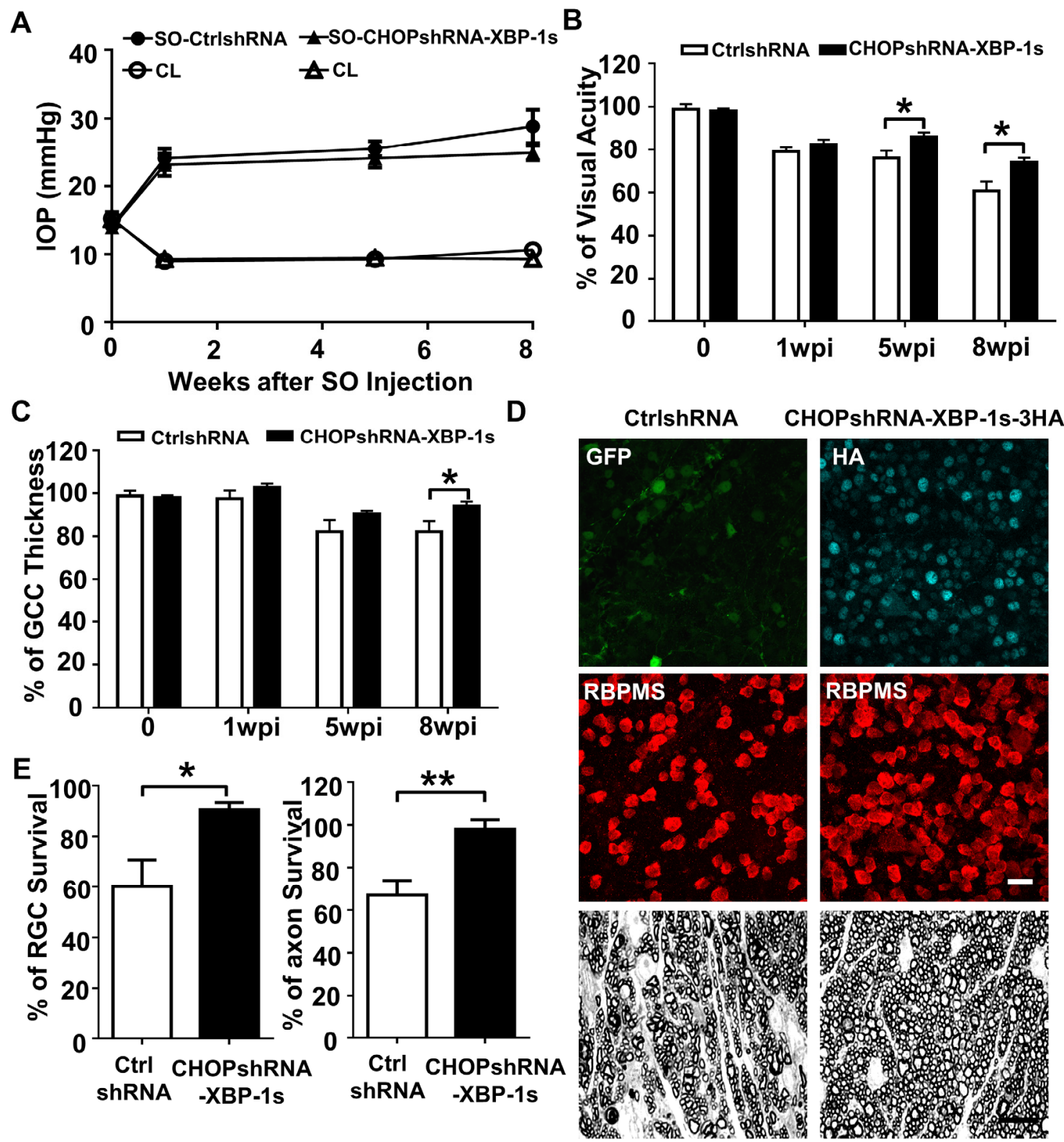

Figure 4

Figure 4. Neuroprotection by ER stress manipulation in SOHU eyes. (A) IOP measurements

272 at different time points after SO injection. AAV-control shRNA-GFP, $n=11$; AAV-CHOP

273 shRNA-XBP-1s-3HA, $n=12$ (B) Visual acuity measured by OKR, represented as percentage of

274 visual acuity in the SO eyes, compared to the CL eyes. $\mathrm{n}=14-15$. Data are presented as means \pm

275 s.e.m, *: p<0.05, Student's t-test. (C) Quantification of GCC thickness measured by OCT,

276 represented as percentage of GCC thickness in the SO eyes, compared to the CL eyes. $n=10$. 
Data are presented as means \pm s.e.m, $*: \mathrm{p}<0.05$, Student's t-test. (D) Upper panel, confocal images of portions of flat-mounted retinas showing control shRNA-GFP positive cells or HA-tag labeled CHOP shRNA-XBP-1s-3HA positive cells; middle panel showing surviving RBPMS positive (red) RGCs at 8wpi. Scale bar, $20 \mu \mathrm{m}$. Lower panel, light microscope images of semithin transverse sections of ON stained with PPD at 8wpi. Scale bar, $10 \mu \mathrm{m}$. (E) Quantification of surviving RGCs $(n=12)$ and surviving axons in $\mathrm{ON}(n=10)$ at $8 \mathrm{wpi}$, represented as percentage of SO eyes compared to the CL eyes. Data are presented as means \pm s.e.m. ${ }^{*} P<0.05, * * P<0.01$; Student's t-test.

One of the disadvantages of many other glaucoma models is that the initial eye injury is irreversible. However, we were able to flush out the SO from the anterior chamber with the aid of normal saline infiltration (Fig. S2A, Movie S5). This procedure lowered the IOP back to normal quickly and stably (Fig. S2B), suggesting that SOHU is a reversible model that can be used to test whether lowering IOP affects degeneration of glaucomatous RGCs or the combination effect with neuroprotection. 


\section{Discussion}

300 relative to pupil size.

A reliable animal glaucoma model that closely mimics the disease in humans is a prerequisite for studies of pathogenetic mechanisms and for selecting efficient neuroprotective treatments for clinical use. In the present study, we applied a highly effective and reproducible method adopted from a clinical secondary glaucoma complication after retina surgery. Injection of SO to the mouse anterior chamber efficiently induces a series of reactions, including pupillary block, blockage of the aqueous humor outflow from anterior chamber, accumulation of aqueous humor in the posterior chamber, closure of the anterior chamber angle, and IOP elevation. These reactions occur without causing overt ocular structural damage or inflammatory responses while simulating glaucomatous changes in human patients in years by inducing progressive RGC and ON degeneration and visual functional deficits within weeks.

SO injection is limited to one eye in each mouse, with the other eye receiving an equivalent volume of normal saline. This serves as a convenient internal control for the surgical procedure and for studies of RGC morphology and function. It is reasonable to conclude that IOP is elevated in the SOHU eyes because of impeded outflow and accumulation of aqueous humor in the posterior segment of the eye, rather than by an aspect of the surgical procedure, such as the cornea wound, inflammation, or TM damage. The relatively small variability in the duration and magnitude of IOP elevation in SOHU eyes after a single injection makes it a simple and reliable ocular hypertension model, which can be explained by the persistence of an SO droplet large 

elevated in the posterior part of the eye, but not in the anterior chamber, which has two advantages: 1) The anterior segments of the experimental eyes are not substantially affected, leaving clear ocular elements that allow easy and reliable assessment of in vivo visual function and morphology; 2) The high IOP of posterior chamber causes pronounced glaucomatous neurodegeneration within 5-8 weeks, which facilitates testing neuroprotectants by allowing any benefit to be detected in a short period of experimental time. One caveat, however, is that SO itself in anterior chamber may blur vision or affect the visual function assays because its optical characteristics differ from those of aqueous humor. These differences may cause early decreases in visual acuity and PERG amplitude at 1wpi, when OCT imaging, which does not depend on the transparency of anterior segment of the eye, shows no significant morphological degeneration. It is also possible that deficits in visual function precede morphological changes, or that there is no proportional relationship between RGC function and RGC morphology, since the visual acuity and PERG amplitude are not always correlated with RGC numbers. An assay of visual function that is unaffected by SO in the anterior chamber and that is more quantitatively related to RGC numbers is needed to resolve the discrepancy definitively. cascade associated with ocular hypertension, but it is not suitable for TM function/deficit studies

340 because it depends on pupillary block. Because of the quick IOP elevation and severe 341 neurodegeneration within a few weeks the SOHU model has features of acute secondary 342 glaucoma in humans, but the extent to which it also more broadly mimics chronic glaucoma in 343 patients needs further investigation. Use of frequent pupil dilation to lower the elevated IOP in 
344 the posterior chamber of the eye would make SOHU a dynamic glaucoma model with IOP

345 fluctuation that is close to the realistic features of primary open angle glaucoma in the clinic.

346

347

348

349

350

351

352

353

354

355

356

As in our previous studies of other optic neuropathy models $(29,34,35)$, we validated that targeting CHOP and XBP-1 together provides significant neuroprotection in the SOHU model. Moreover, we successfully used a single AAV vector to inhibit and activate different molecules in RGCs, indicating a promising novel gene therapy strategy. The present proof-of-concept application of the SOHU model demonstrates its usefulness for selecting neuroprotectants and testing the effectiveness of neuroprotective therapies in vivo, including in larger animal species. One interesting implication of the current study is that AAV itself may provide neuroprotection through inflammation or activation of other protective pathways; determining the mechanisms warrants further investigation.

post-operative glaucoma. It is straightforward, does not require special equipment or repeat injections, and may be applicable to a range of animal species with only minor modifications.

We also demonstrated that it is easily reversible by removing SO from the anterior chamber and that is useful for screening neuroprotective therapies in vivo. Therefore we report this simple, convenient, effective, reproducible, and reversible mouse model that generates stable, robust IOP elevation and significant neurodegeneration within weeks with the hopes that it will standardize assessment of the pathogenesis of ocular hypertension-induced glaucoma and facilitate selection of neuroprotectants for glaucoma. 


\section{Methods}

Mice. C57BL/6J WT mice were purchased from Jackson Laboratories (Bar Harbor, Maine). For all surgical and treatment comparisons, control and treatment groups were prepared together in single cohorts, and the experiment repeated at least twice. All experimental procedures were performed in compliance with animal protocols approved by the IACUC at Stanford University School of Medicine.

Induction of IOP elevation by intracameral injection of SO. Mice were anesthetized by an intraperitoneal injection of Avertin $(0.3 \mathrm{mg} / \mathrm{g})$ instead of ketamine/xylazine to avoid pupil dilation. The mice were then placed in a lateral position on a surgery platform. Prior to injection, one drop of $0.5 \%$ proparacaine hydrochloride (Akorn, Somerset, New Jersey) was applied to the cornea to reduce its sensitivity during the procedure. A $32 \mathrm{G}$ needle was tunneled through the layers of the cornea at the superotemporal side close to the limbus to reach the anterior chamber without injuring lens or iris. Following this entry, about $2 \mu 1$ silicone oil (1,000 mPa.s, Silikon, Alcon Laboratories, Fort Worth, Texas) were injected slowly into the anterior chamber using a homemade sterile glass micropipette, until the oil droplet expanded to cover most areas of the iris. The micropipette was held in place for 30 seconds before withdrawing it slowly. After the injection, the upper eyelid was gently massaged to close the corneal incision to minimize SO leakage, and veterinary antibiotic ointment (BNP ophthalmic ointment, Vetropolycin, Dechra, Overland Park, Kansas) was applied to the surface of the injected eye. The contralateral control eyes received $2 \mu 1$ normal saline to the anterior chamber. During the whole procedure, artificial tears (Systane Ultra Lubricant Eye Drops, Alcon Laboratories, Fort Worth, Texas) were applied 
to keep the cornea moist. Rare, some mice showed corneal opacity associated with band-shaped degeneration or neovascularization, and were excluded from further analysis.

Removing SO from the anterior chamber. The oil droplet was removed from the anterior placed in a lateral position on a surgery platform. Prior to injection, one drop of $0.5 \%$ proparacaine hydrochloride (Akorn, Somerset, New Jersey) was applied to the cornea to reduce its sensitivity during the procedure. Then two corneal tunnel incisions were made using a $32 \mathrm{G}$ needle: one tunnel incision superior and one tunnel incision inferior to the center of the cornea, each at the edge of the oil droplet. A $33 \mathrm{G}$ needle attached to an elevated balanced salt solution plus (BSS Plus, Alcon Laboratories, Ft. Worth, Texas) drip (110 cm $\mathrm{H}_{2} \mathrm{O}$ height, equal to $81 \mathrm{mmHg}$ ) was inserted through the superior corneal incision to flow BSS into anterior chamber to maintain its volume. At the same time, another $33 \mathrm{G}$ needle attached to a $1 \mathrm{~mL}$ syringe with the plunger removed, was inserted through the inferior tunnel incision to allow SO outflow. After removing the oil, a small air bubble was injected by a glass micropipette into anterior chamber to maintain the volume of anterior chamber and temporarily seal the corneal incision. Veterinary antibiotic ointment (BNP ophthalmic ointment) was applied to the surface of the eye.

IOP measurement. The IOP of both eyes was monitored once weekly until 8 weeks after SO

409 injection using the TonoLab tonometer (Colonial Medical Supply, Espoo, Finland) according to 410 product instructions. Briefly, mice were anesthetized with a sustained flow of isoflurane $(3 \%$ 411 isoflurane at $2 \mathrm{~L} /$ minute mixed with oxygen) delivered to the nose by a special rodent nose cone 412 (Xenotec, Inc., Rolla, Missouri), which left the eyes exposed for IOP measurement. The 
TonoLab tonometer takes five measurements, eliminates high and low readings and generates an average. We considered this machine-generated average as one reading. Three machinegenerated readings were obtained from each eye, and the mean was calculated to determine the IOP. During this procedure, artificial tears were applied to keep the cornea moist.

Pattern electroretinogram (PERG) recording. Mice were anesthetized by xylazine and ketamine based on their body weight $(0.01 \mathrm{mg}$ xylazine $/ \mathrm{g}+0.08 \mathrm{mg}$ ketamine $/ \mathrm{g})$. PERG recording of both eyes was performed at the same time with the Miami PERG system (Intelligent Hearing Systems, Miami, FL) according to published protocol(45). Briefly, mice were placed on a feedback-controlled heating pad (TCAT-2LV, Physitemp Instruments Inc., Clifton, New Jersey) to maintain animal core temperature at $37^{\circ} \mathrm{C}$. A small lubricant eye drop (Systane Ultra Lubricant Eye Drops, Alcon Laboratories, Ft. Worth, Texas) was applied before recording to prevent corneal dryness. The reference electrode was placed subcutaneously on the back of the head between the two ears and the ground electrode was placed at the root of the tail. The active steel needle electrode was placed subcutaneously on the snout for the simultaneous acquisition of left and right eye responses. Two $14 \mathrm{~cm} \times 14 \mathrm{~cm}$ LED-based stimulators were placed in front so that the center of each screen was $10 \mathrm{~cm}$ from each eye. The pattern remained at a contrast of $85 \%$ and a luminance of $800 \mathrm{~cd} / \mathrm{m}^{2}$, and consisted of four cycles of black-gray elements, with a spatial frequency of $0.052 \mathrm{c} / \mathrm{d}$. Upon stimulation, the independent PERG signals were recorded from the snout and simultaneously by asynchronous binocular acquisition. With each trace recording up to $1020 \mathrm{~ms}$, two consecutive recordings of 200 traces were averaged to achieve one readout. The first positive peak in the waveform was designated as P1 (typically around 100ms) and the second negative peak as N2 (typically around 205ms). The amplitude was measured 
from P1 to N2. The mean of the P1-N2 amplitude in the injured eye was compared to that in the contralateral control eye to yield a percentage of amplitude change. The investigators who measured the amplitudes were masked to the treatment of the samples.

Spectral-domain optical coherence tomography (SD-OCT) imaging. After the mice were anesthetized, pupils were dilated by applying 1\% tropicamide sterile ophthalmic solution (Akorn, Somerset, New Jersey), and a customized $+10 \mathrm{D}$ contact lens $(3.0 \mathrm{~mm}$ diameter, $1.6 \mathrm{~mm} \mathrm{BC}$, PMMA clear, Advanced Vision Technologies) applied to the dilated pupil. The retina fundus images were captured with the Heidelberg Spectralis SLO/OCT system (Heidelberg Engineering, Germany) equipped with an 870nm infrared wavelength light source and a $30^{\circ}$ lens (Heidelberg Engineering). The OCT scanner has $7 \mu \mathrm{m}$ optical axial resolution, $3.5 \mu \mathrm{m}$ digital resolution, and $1.8 \mathrm{~mm}$ scan depth at $40 \mathrm{kHz}$ scan rate. The mouse retina was scanned with the ring scan mode centered by the optic nerve head at 100 frames average under high-resolution mode (each B-scan consisted of 1536 A scans). The GCC includes retinal nerve fiber layer (RNFL), ganglion cell layer (GCL) and inner plexiform layer (IPL). The average thickness of GCC around the optic nerve head was measured manually with the aid of Heidelberg software. The mean of the GCC thickness in the injured retina was compared to that in the contralateral control retina to yield a percentage of GCC thickness value. The investigators who measured the thickness of GCC were masked to the treatment of the samples.

OKR measurement. To measure the spatial vision using the opto-kinetic response (OKR), mice were placed unrestrained on a platform in the center of four 17-inch LCD computer monitors (Dell, Phoenix, AZ), with a video camera above the platform to capture the movement of the 
mouse. A rotating cylinder with vertical sine wave grating was computed and projected to the four monitors by OptoMotry software (CerebralMechanics Inc., Lethbridge, Alberta, Canada). The sine wave grating, consisting of black (mean luminance $0.22 \mathrm{~cd} / \mathrm{m} 2$ ) and white (mean luminance $152.13 \mathrm{~cd} / \mathrm{m} 2$ ) at $100 \%$ contrast and 12 degree/second, provides a virtual-reality environment to measure the spatial acuity of left eye when rotates clockwise and right eye when it rotates counterclockwise. Initially, the monitors were covered with gray so that the mouse calmed down and stopped moving, then the gray was switched to a low spatial frequency $(0.1$ cycle/degree) for five seconds, during which the mouse was assessed for whether the head turned to track the grating. The short time frame of assessment ensures that the mice did not adapt to the stimulus, which would lead to false readouts. When the mouse was determined to be capable of tracking the grating, the spatial frequency was increased repeatedly until the maximum frequency was identified and recorded. At each time point, the maximum frequency of the experimental eye was compared to that of the contralateral eye. The mice were tested in the morning and the investigator who judged the OKR was masked to the treatment of mice.

Statistical analyses. GraphPad Prism 6 was used to generate graphs and for statistical analyses. Data are presented as means \pm s.e.m. Student's t-test was used for two groups comparison and One-way ANOVA with post hoc test was used for multiple comparisons.

Additional experimental details are provided in SI Appendix, including Immunohistochemistry of whole-mount retina and RGC counting, $\mathrm{ON}$ semi-thin sections and quantification of surviving axons, AAV production, Intravitreal injection. 


\section{Author contributions}

484 Y.H. J.Z., L.L. and H.H. designed the experiments. J.Z., L.L., H.H., H.C.W., P.Z., L.L. and R.D.

485 performed the experiments and analyzed the data. P.H.L, V.B.M., Y.S., S.L., M.Z. and J.L.G.

486 helped with discussion about clinical secondary glaucoma and animal models. Y.H. J.Z., L.L.,

487 and H.H. prepared the manuscript.

488

489 


\section{Acknowledgements}

491 We thank Drs. Alan Tessler and Fang Fang for critically reading the manuscript. We also 492 appreciate the help from Gang Jiang and Niannian Liu in making schematic diagrams in Fig. 1A. 493 Y.H. is supported by NIH grants EY024932, EY023295 and EY028106 and grants from 494 BrightFocus Foundation, Glaucoma Research Foundation, National Multiple Sclerosis Society 495 and William \& Mary Greve Special Scholar Award from Research to Prevent Blindness. 496 Portions of this work were supported by NIH grants EY026766 and EY027261 to J.L.G and NIH 497 grants EY-25295, K08-EY022058, VA CX001298, Ziegler Foundation for the Blind to Y.S, who 498 is a Stanford Child Health Research Institute Laurie Kraus Lacob Faculty Scholar. H.C.W. is 499 supported by NIH T32 Postdoctoral Fellowship (NEI T32 EY027816). We are grateful for an 500 unrestricted grant from Research to Prevent Blindness and NEI P30-026877 to the Department of 501 Ophthalmology. The authors have declared that no conflict of interest exists. 


\section{References}

1. Tham YC, et al. (2014) Global prevalence of glaucoma and projections of glaucoma burden through 2040: a systematic review and meta-analysis. Ophthalmology 121(11):2081-2090.

2. Varma R, Lee PP, Goldberg I, \& Kotak S (2011) An assessment of the health and economic burdens of glaucoma. American journal of ophthalmology 152(4):515-522.

3. $\quad$ Quigley HA (1993) Open-angle glaucoma. N Engl J Med 328(15):1097-1106.

4. Quigley HA, et al. (1995) Retinal ganglion cell death in experimental glaucoma and after axotomy occurs by apoptosis. Invest Ophthalmol Vis Sci 36(5):774-786.

5. Libby RT, et al. (2005) Susceptibility to neurodegeneration in a glaucoma is modified by Bax gene dosage. PLoS Genet 1(1):17-26.

6. Howell GR, et al. (2007) Axons of retinal ganglion cells are insulted in the optic nerve early in DBA/2J glaucoma. J Cell Biol 179(7):1523-1537.

7. Weinreb RN \& Khaw PT (2004) Primary open-angle glaucoma. Lancet 363(9422):1711-1720.

8. Calkins DJ (2012) Critical pathogenic events underlying progression of neurodegeneration in glaucoma. Prog Retin Eye Res 31(6):702-719.

9. Burgoyne CF (2011) A biomechanical paradigm for axonal insult within the optic nerve head in aging and glaucoma. Experimental eye research 93(2):120-132.

10. Nickells RW, Howell GR, Soto I, \& John SW (2012) Under pressure: cellular and molecular responses during glaucoma, a common neurodegeneration with axonopathy. Annu Rev Neurosci 35:153-179.

11. Jonas JB, et al. (2017) Glaucoma. Lancet 390(10108):2183-2193.

12. Singh K \& Shrivastava A (2009) Intraocular pressure fluctuations: how much do they matter? Curr Opin Ophthalmol 20(2):84-87.

13. Anonymous (2000) The Advanced Glaucoma Intervention Study (AGIS): 7. The relationship between control of intraocular pressure and visual field deterioration. The AGIS Investigators. American journal of ophthalmology 130(4):429-440.

14. Heijl A, Leske MC, Bengtsson B, Hyman L, \& Hussein M (2002) Reduction of intraocular pressure and glaucoma progression: results from the Early Manifest Glaucoma Trial. Arch Ophthalmol 120(10):12681279.

15. Lichter PR, et al. (2001) Interim clinical outcomes in the Collaborative Initial Glaucoma Treatment Study comparing initial treatment randomized to medications or surgery. Ophthalmology 108(11):1943-1953.

16. Varma R, Peeples P, Walt JG, \& Bramley TJ (2008) Disease progression and the need for neuroprotection in glaucoma management. The American journal of managed care 14(1 Suppl):S15-19.

17. Pang IH \& Clark AF (2007) Rodent models for glaucoma retinopathy and optic neuropathy. Journal of glaucoma 16(5):483-505.

18. Morrison JC, Johnson E, \& Cepurna WO (2008) Rat models for glaucoma research. Prog Brain Res 173:285-301.

19. McKinnon SJ, Schlamp CL, \& Nickells RW (2009) Mouse models of retinal ganglion cell death and glaucoma. Experimental eye research 88(4):816-824.

20. Chen S \& Zhang X (2015) The Rodent Model of Glaucoma and Its Implications. Asia Pac J Ophthalmol (Phila) 4(4):236-241.

21. Aihara M, Lindsey JD, \& Weinreb RN (2003) Experimental mouse ocular hypertension: establishment of the model. Invest Ophthalmol Vis Sci 44(10):4314-4320.

22. Grozdanic SD, et al. (2003) Laser-induced mouse model of chronic ocular hypertension. Invest Ophthalmol Vis Sci 44(10):4337-4346.

23. Yun H, et al. (2014) A laser-induced mouse model with long-term intraocular pressure elevation. PLoS One 9(9):e107446.

24. Sappington RM, Carlson BJ, Crish SD, \& Calkins DJ (2010) The microbead occlusion model: a paradigm for induced ocular hypertension in rats and mice. Invest Ophthalmol Vis Sci 51(1):207-216.

25. Chen H, et al. (2011) Optic neuropathy due to microbead-induced elevated intraocular pressure in the mouse. Invest Ophthalmol Vis Sci 52(1):36-44.

26. Cone FE, Gelman SE, Son JL, Pease ME, \& Quigley HA (2010) Differential susceptibility to experimental glaucoma among 3 mouse strains using bead and viscoelastic injection. Experimental eye research 91(3):415-424. 
27. Samsel PA, Kisiswa L, Erichsen JT, Cross SD, \& Morgan JE (2011) A novel method for the induction of experimental glaucoma using magnetic microspheres. Invest Ophthalmol Vis Sci 52(3):1671-1675.

28. Ito YA, Belforte N, Cueva Vargas JL, \& Di Polo A (2016) A Magnetic Microbead Occlusion Model to Induce Ocular Hypertension-Dependent Glaucoma in Mice. Journal of visualized experiments : JoVE (109): 53731.

29. Yang L, et al. (2016) Rescue of Glaucomatous Neurodegeneration by Differentially Modulating Neuronal Endoplasmic Reticulum Stress Molecules. J Neurosci 36(21):5891-5903.

30. Ichhpujani P, Jindal A, \& Jay Katz L (2009) Silicone oil induced glaucoma: a review. Graefe's archive for clinical and experimental ophthalmology $=$ Albrecht von Graefes Archiv fur klinische und experimentelle Ophthalmologie 247(12):1585-1593.

31. Kornmann HL \& Gedde SJ (2016) Glaucoma management after vitreoretinal surgeries. Curr Opin Ophthalmol 27(2):125-131.

32. Ron D \& Walter P (2007) Signal integration in the endoplasmic reticulum unfolded protein response. Nat Rev Mol Cell Biol 8(7):519-529.

33. Wang S \& Kaufman RJ (2012) The impact of the unfolded protein response on human disease. J Cell Biol 197(7):857-867.

34. Hu Y, et al. (2012) Differential effects of unfolded protein response pathways on axon injury-induced death of retinal ganglion cells. Neuron 73(3):445-452.

35. Huang $\mathrm{H}$, et al. (2017) Neuroprotection by eIF2alpha-CHOP inhibition and XBP-1 activation in EAE/optic neuritiss. Cell death \& disease 8(7):e2936.

36. Nakano N, et al. (2011) Longitudinal and simultaneous imaging of retinal ganglion cells and inner retinal layers in a mouse model of glaucoma induced by N-methyl-D-aspartate. Invest Ophthalmol Vis Sci 52(12):8754-8762.

37. Prusky GT, Alam NM, Beekman S, \& Douglas RM (2004) Rapid quantification of adult and developing mouse spatial vision using a virtual optomotor system. Invest Ophthalmol Vis Sci 45(12):4611-4616.

38. Douglas RM, et al. (2005) Independent visual threshold measurements in the two eyes of freely moving rats and mice using a virtual-reality optokinetic system. Vis Neurosci 22(5):677-684.

39. Porciatti V (2015) Electrophysiological assessment of retinal ganglion cell function. Experimental eye research 141:164-170.

40. Balcer LJ, Miller DH, Reingold SC, \& Cohen JA (2015) Vision and vision-related outcome measures in multiple sclerosis. Brain 138(Pt 1):11-27.

41. Aktas O, Albrecht P, \& Hartung HP (2016) Optic neuritis as a phase 2 paradigm for neuroprotection therapies of multiple sclerosis: update on current trials and perspectives. Current opinion in neurology 29(3):199-204.

42. Costello F, et al. (2006) Quantifying axonal loss after optic neuritis with optical coherence tomography. Annals of neurology 59(6):963-969.

43. Douglas RM, Neve A, Quittenbaum JP, Alam NM, \& Prusky GT (2006) Perception of visual motion coherence by rats and mice. Vision research 46(18):2842-2847.

44. Burroughs SL, Kaja S, \& Koulen P (2011) Quantification of deficits in spatial visual function of mouse models for glaucoma. Invest Ophthalmol Vis Sci 52(6):3654-3659.

45. Chou TH, Bohorquez J, Toft-Nielsen J, Ozdamar O, \& Porciatti V (2014) Robust mouse pattern electroretinograms derived simultaneously from each eye using a common snout electrode. Invest Ophthalmol Vis Sci 55(4):2469-2475. 


\title{
Supplementary Information for
}

2

\section{Silicone Oil-Induced Ocular Hypertension in Mouse Models Glaucomatous Neurodegeneration and Neuroprotection}

\author{
Jie Zhang1,2,†, Liang Li ${ }^{1, \dagger}$, Haoliang Huang${ }^{1}$, Hannah C. Webber ${ }^{1}$, Pei Zhuang ${ }^{1}$, Liang Liu ${ }^{1}$, \\ Roopa Dalal ${ }^{1}$, Peter H. Tang,3, Vinit B. Mahajan ${ }^{1,3}$, Yang Sun ${ }^{1,3}$, Shaohua Li $^{2}$, Mingchang \\ Zhang $^{2}$, Jeffrey Louis Goldberg ${ }^{1}$ and Yang $\mathrm{Hu}^{1, *}$ \\ Corresponding author: Yang $\mathrm{Hu}$ \\ Email: huyang@stanford.edu
}

This PDF file includes:

Supplementary Experimental Methods

Fig. S1. SO itself does not cause glaucomatous degeneration.

Fig. S2. SOHU is reversible by SO removal.

Other supplementary materials for this manuscript include the following:

Movie S1. Intracameral SO injection.

Movie S2. Dye migration from vitreous chamber to anterior chamber in naïve eyes.

Movie S3. Dye migration blocked in SOHU eyes.

Movie S4. SO droplet flows away from pupil after dilation.

Movie S5. SO removal from SOHU eyes. 


\section{Experimental Methods}

Immunohistochemistry of whole-mount retina and RGC counting. After transcardiac perfusion with 4\% PFA in PBS, the eyes were dissected out, post-fixed with 4\% PFA for 2 hours, at room temperature, and cryoprotected in $30 \%$ sucrose at $4{ }^{\circ} \mathrm{C}$ overnight. Retinas were dissected out and washed extensively in PBS before blocking in staining buffer $(10 \%$ normal goat serum and 2\% Triton X-100 in PBS) for half an hour. RBPMS guinea pig antibody made at ProSci, California according to publications $(1,2)$ and used at 1:4000, and rat HA (clone 3F10, 1:200, Roche) were diluted in the same staining buffer. Floating retinas were incubated with primary antibodies overnight at $4{ }^{\circ} \mathrm{C}$ and washed 3 times for 30 minutes each with PBS. Secondary antibodies (Cy2 or Cy3) were then applied (1:200-400; Jackson ImmunoResearch, West Grove, Pennsylvania) and incubated for 1 hour at room temperature. Retinas were again washed 3 times for 30 minutes each with PBS before a cover slip was attached with Fluoromount-G (SouthernBiotech, Birmingham, Alabama). For RGC counting, whole-mount retinas were immunostained with the RBPMS antibody, 6-9 fields randomly sampled from peripheral regions of each retina using 40x lens with a Zeiss M2 epifluorescence microscope, and $\mathrm{RBPMS}^{+}$RGCs counted by Volocity software (Quorum Technologies). The percentage of RGC survival was calculated as the ratio of surviving RGC numbers in injured eyes compared to contralateral uninjured eyes. The investigators who counted the cells were masked to the treatment of the samples.

ON semi-thin sections and quantification of surviving axons. After mice were perfused through the heart with ice cold 4\% paraformaldehyde (PFA) in PBS, the ON was exposed by removing the brain and post-fixed in situ using 2\% glutaraldehyde/ $2 \%$ PFA in $0.1 \mathrm{M}$ PB for 4 hours on ice. Samples were then washed with 0.1M PB 3 times, 10 minutes each wash. The ONs 
were then carefully dissected out and rinsed with $0.1 \mathrm{M}$ PB 3 times, 10 minutes each wash. They were then incubated in $1 \%$ osmium tetroxide in $0.1 \mathrm{M} \mathrm{PB}$ for 1 hour at room temperature followed by washing with $0.1 \mathrm{M}$ PB for 10 minutes and water for 5 minutes. ONs were next dehydrated through a series of graded ethanol (50\% to 100\%), rinsed twice with propylene oxide (P.O.), 3 minutes each rinse, and transferred to medium containing 50\% EMbed 812 / 50\% P.O. overnight. The next day, the medium was changed to a 2:1 ratio of EMbed 812/P.O. ONs remained in this mixture overnight, then were transferred to $100 \%$ EMbed 812 on a rotator for another 6 hours, embedded in a mold filled with $100 \%$ EMbed 812 and incubated at $60^{\circ} \mathrm{C}$ overnight. Semi-thin sections $(1 \mu \mathrm{m})$ were cut on an ultramicrotome (EM UC7, Leica, Wetzlar, Germany) and collected $2 \mathrm{~mm}$ distal to the eye. The semi-thin sections were attached to glass slides and stained with 1\% para-phenylenediamine (PPD) in methanol: isopropanol (1:1) for 35 minutes. After rinsing 3 times with methanol: isopropanol (1:1), coverslips were applied with Permount Mounting Medium (Electron Microscopy Sciences, Hatfield, Pennsylvania). PPD stains all myelin sheaths, but darkly stains the axoplasm only of degenerating axons, which allows us to differentiate surviving axons from degenerating axons(3). Four sections of each ON were imaged through a 100x lens of a Zeiss M2 epifluorescence microscope to cover the entire area of the ON without overlap. Two areas of $21.4 \mu \mathrm{m}$ X $29.1 \mu \mathrm{m}$ were cropped from the center of each image, and the surviving axons within the designated areas were counted manually. After counting all the images taken from a single nerve, the mean of the surviving axon number was calculated for each ON. The mean of the surviving axon number in the injured ON was compared to that in the contralateral control ON to yield a percentage of axon survival value. The investigators who counted the axons were masked to the treatment of the samples. 
AAV production. The detailed procedure has been described previously $(4,5)$. Briefly, AAV plasmids containing miRNA based uBC promoter-driven CHOP shRNA and XBP-1s-3HA were co-transfected with pAAV2 (pACG2)-RC triple mutant (Y444, 500, 730F)(6-8) and the pHelper plasmid (Stratagene) into HEK293T cells. 72 hours after transfection, the cells were lysed to release the viral particles, which were precipitated by $40 \%$ polyethylene glycol and purified by two rounds of cesium chloride density gradient centrifugation. The virus bands were taken out for dialysis in a MWCO 7000 Slide-A -LYZER cassette (Pierce) overnight at $4^{\circ} \mathrm{C}$. The AAV titers were determined by real-time PCR and diluted to $1.5 \times 10^{12}$ vector genome $(\mathrm{vg}) / \mathrm{ml}$. Four copies of different mouse CHOP RNAi sequences identified from the RNAi Consortium (5'ATtTCATCTGAGGACAGGACC-3'; [ 5'-CATAGAACTCTGACTGGAATC-3'; : 5'TTCCGTTTCCTAGTTCTTCCT-3'; 5'-CGATTTCCTGCTTGAG CCGCT-3') with modified miR-155 stem-loops and GFP were driven by the $\mathrm{uBC}$ promoter in an AAV backbone(9), a gift from Dr. Kevin Park. XBP-1s-3HA was subcloned into this vector by replacing GFP.

Intravitreal injection. These procedures have been described previously(4, 5). Briefly, mice were anesthetized by xylazine and ketamine based on their body weight $(0.01 \mathrm{mg}$ xylazine/g+0.08 mg ketamine/g). For each AAV intravitreal injection, a micropipette was inserted into the peripheral retina of adult mice just behind the ora serrata, and advanced into the vitreous chamber so as to avoid damage to the lens. Approximately $2 \mu 1$ of the vitreous was removed before injection of $2 \mu \mathrm{AAV}$ into the vitreous chamber. Intravitreal SO injection was the same procedure. For intravitreal dye injection, DiI solution (ThermoFisher Scientific, V22885) was injected into posterior chamber through the point directly behind the limbus (beneath the iris) to demonstrate aqueous humor migration. 

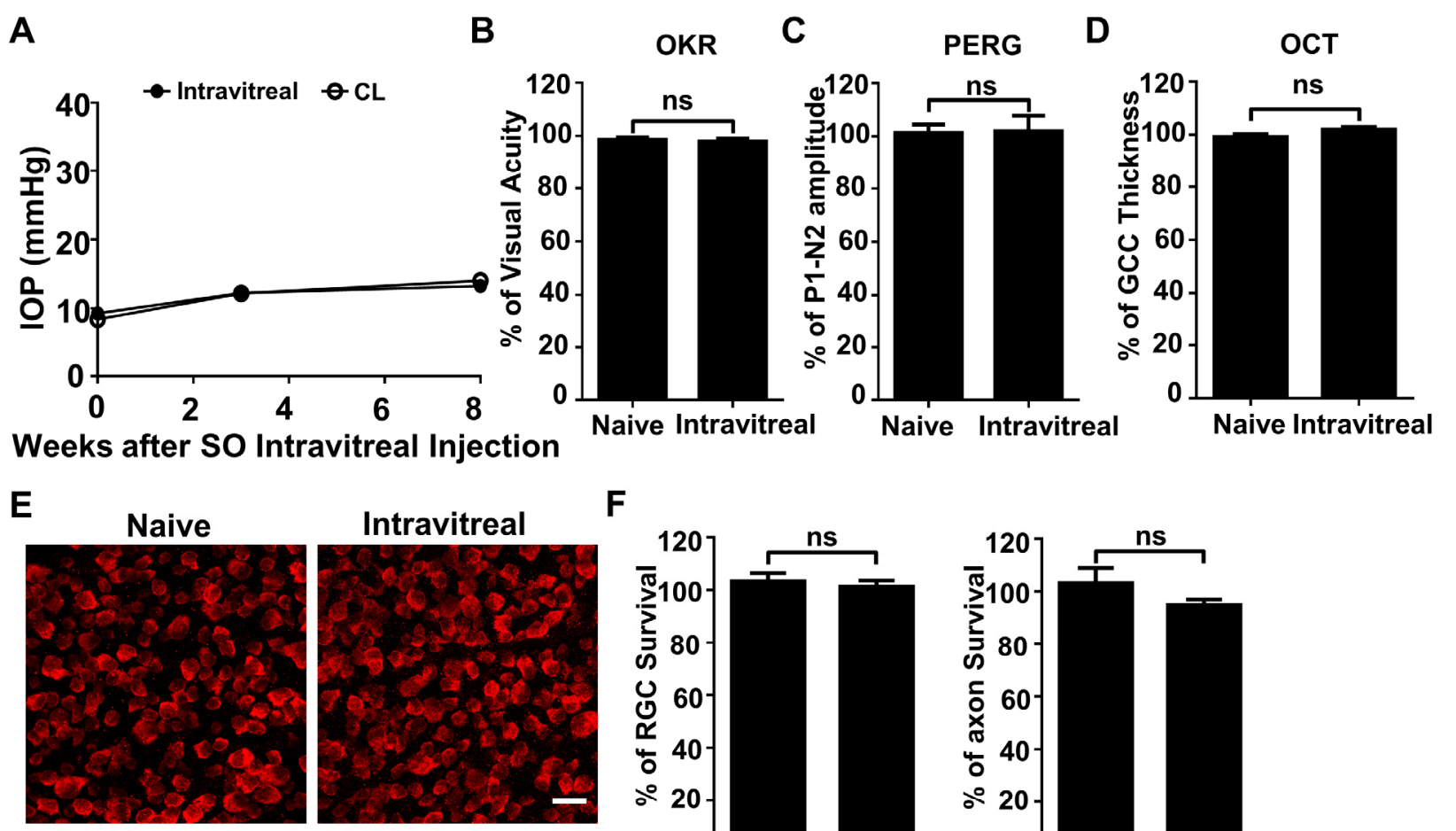

F
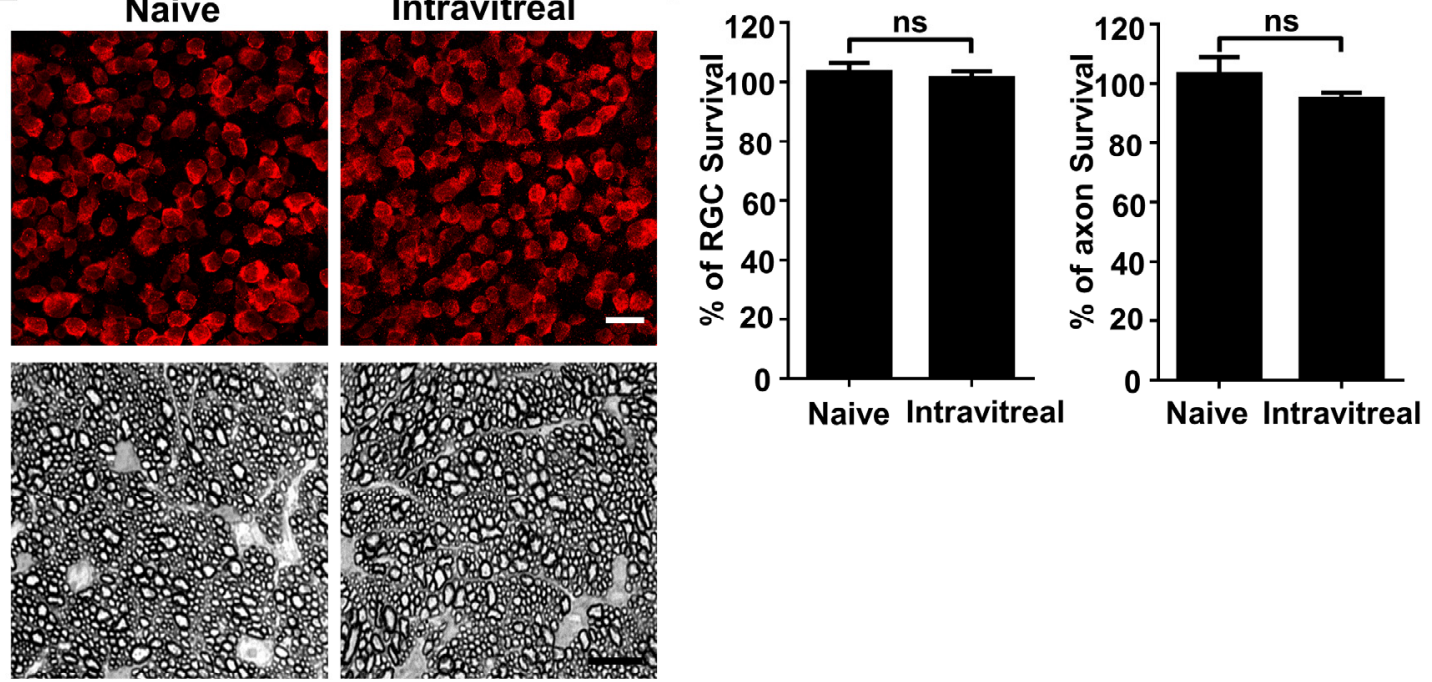

G
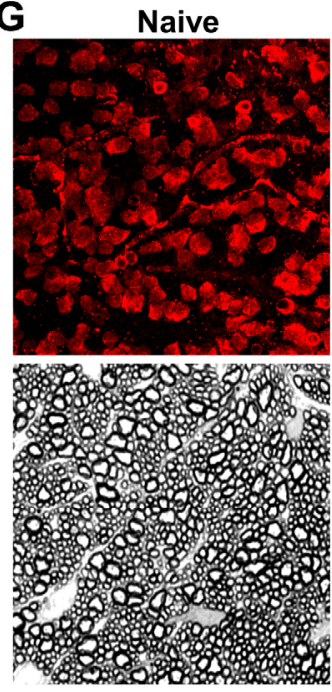
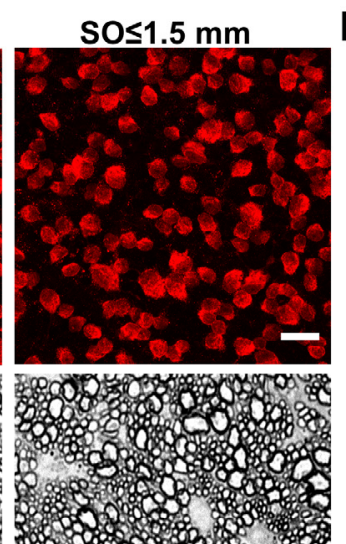

H

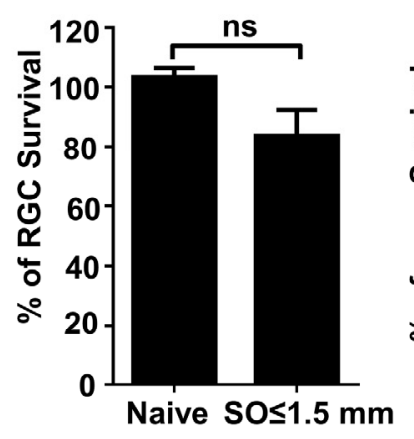

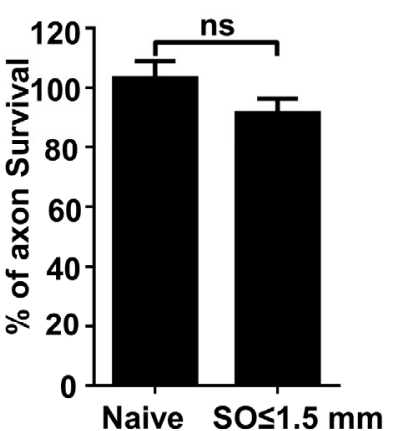


Figure S1. SO itself does not cause glaucomatous degeneration. (A) IOP measurements at different time points after intravitreal SO injection. $\mathrm{n}=15$. (B) Visual acuity measured by OKR, represented as percentage of visual acuity in the SO eyes, compared to the CL eyes. $n=13-15$. (C) Quantification of P1-N2 amplitude of PERG, represented as percentage of P1-N2 amplitude in the SO eyes, compared to the CL eyes. $n=12-15$. (D) Quantification of GCC thickness measured by OCT, represented as percentage of GCC thickness in the SO eyes, compared to the CL eyes. $\mathrm{n}=11-13$. (E) Upper panel, confocal images of portions of flat-mounted retinas showing surviving RBPMS-positive (red) RGCs at 8wpi after intravitreal SO injection and contralateral naive eye. Scale bar, $20 \mu \mathrm{m}$. Lower panel, light microscope images of semi-thin transverse sections of ON stained with PPD at 8wpi after intravitreal SO injection and contralateral naive eye. Scale bar, $10 \mu \mathrm{m}$. (F) Quantification of surviving RGCs ( $n=10)$ and surviving axons in ON $(n=10)$ at 8 wpi after intravitreal SO injection, represented as percentage of SO eyes compared to the CL eyes. Data are presented as means \pm s.e.m, Student t-test. (G) Upper panel, confocal images of portion of flat-mounted retinas showing surviving RBPMS positive (red) RGCs at 8 wpi after intracameral SO injection (small size of SO droplet, $\leq 1.5 \mathrm{~mm}$ ) and contralateral naive eye. Scale bar, $20 \mu \mathrm{m}$. Lower panel, light microscope images of semi-thin transverse sections of ON stained with PPD at 8wpi after intracameral SO injection and contralateral naive eye. Scale bar, $10 \mu \mathrm{m}$. (E) Quantification of surviving RGCs $(n=12)$ and surviving axons in ON $(n=13)$ at 8wpi, represented as percentage of SO eyes compared to the CL eyes. Data are presented as means \pm s.e.m, Student t-test. 
A
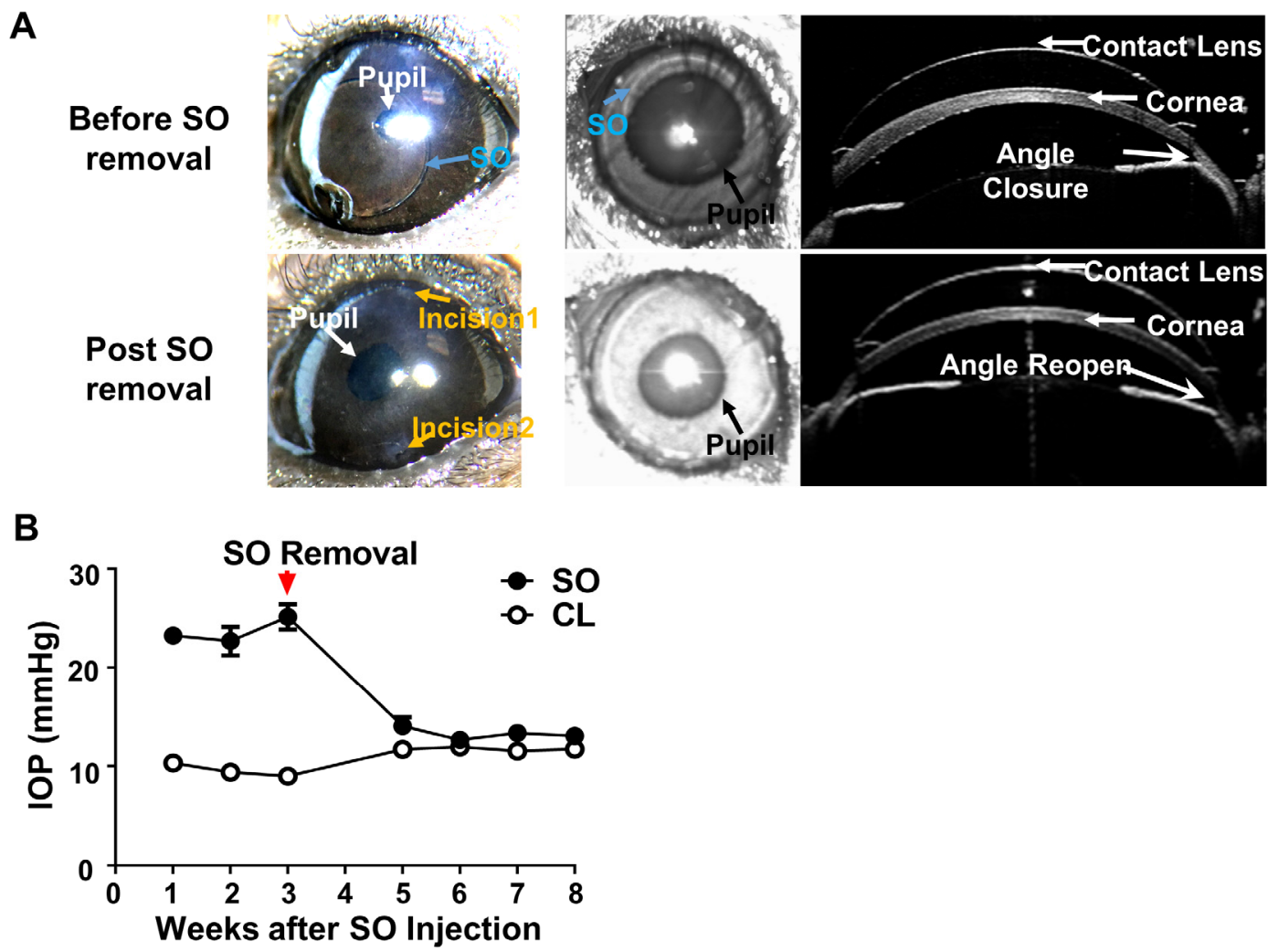

121 Figure S2. SOHU is reversible by SO removal. (A) Representative images of SOHU eyes

122 before and after SO removal, and anterior chamber OCT images in living animals showing the

123 relative size of SO droplet to pupil and the corresponding closure or opening of the anterior

124 chamber angle before and after SO removal. (B) IOP measurements before and after SO removal

125 at different time points. $\mathrm{n}=16$. 


\section{Movie legends}

132 Movie S1. Intracameral SO injection. Demonstration of the anterior chamber SO injection

133 with a glass pippet and the SO droplet formation on top of iris to block pupil. injected into the posterior chamber of the naïve eye and migrated into the anterior chamber.

Movie S3. Dye migration blocked in SOHU eyes. DiI injected into the posterior chamber of the SOHU eye and there was no DiI detected in the anterior chamber. chamber.

Movie S5. SO removal from SOHU eyes. To remove SO from the anterior chamber, one needle glass pippet was used to suck away the SO from the anterior chamber. 


\section{Supplemental References}

150 1. J. M. Kwong, J. Caprioli, N. Piri, RNA binding protein with multiple splicing: a new marker for retinal ganglion cells. Invest Ophthalmol Vis Sci 51, 1052-1058 (2010). A. R. Rodriguez, L. P. de Sevilla Muller, N. C. Brecha, The RNA binding protein RBPMS is a selective marker of ganglion cells in the mammalian retina. The Journal of comparative neurology 522, 1411-1443 (2014). biomethods. Research methods for mutant mice series (CRC Press, Boca Raton, 2002), pp. 366 p.

4. Y. Hu et al., Differential effects of unfolded protein response pathways on axon injuryinduced death of retinal ganglion cells. Neuron 73, 445-452 (2012).

160

5. L. Yang et al., The mTORC1 effectors S6K1 and 4E-BP play different roles in CNS axon

161

162 regeneration. Nature communications 5, 5416 (2014).

6. H. Petrs-Silva et al., Novel properties of tyrosine-mutant AAV2 vectors in the mouse retina. Mol Ther 19, 293-301 (2011).

163

164

7. L. N. Wang et al., Pristimerin enhances recombinant adeno-associated virus vectormediated transgene expression in human cell lines in vitro and murine hepatocytes in vivo. Journal of integrative medicine 12, 20-34 (2014).

166

167

8. Y. H. Zhang et al., Cytotoxic genes from traditional Chinese medicine inhibit tumor

168 growth both in vitro and in vivo. Journal of integrative medicine 12, 483-494 (2014).

169

9. K. H. Chung et al., Polycistronic RNA polymerase II expression vectors for RNA interference based on BIC/miR-155. Nucleic acids research 34, e53 (2006). 